\title{
SOLVABILITY OF DIFFERENTIAL EQUATIONS WITH LINEAR COEFFICIENTS OF NILPOTENT TYPE
}

\author{
RAINER FELIX
}

\begin{abstract}
Let $L$ be the vector field on $\mathbf{R}^{n}$ associated with a real nilpotent $(n \times n)$-matrix. It is shown that $L$ regarded as a differential operator defines a surjective mapping of the space $\mathscr{S}^{\prime}$ of tempered distributions onto itself; i.e. $L \mathscr{S}^{\prime}\left(\mathbf{R}^{n}\right)=\mathscr{S}^{\prime}\left(\mathbf{R}^{n}\right)$. Replacing $\mathscr{S}^{\prime}$ by the space $\mathscr{D}^{\prime}$ of ordinary distributions, this is not true in general.
\end{abstract}

1. Introduction. Let $L$ be the infinitesimal transformation on $\mathbf{R}^{n}$ associated with a real $(n \times n)$-matrix $X$; i.e. $L$ is given by

$$
L \varphi(x)=\left.\frac{d}{d t} \varphi\left(e^{-t X} x\right)\right|_{t=0}=-\sum_{i=1}^{n}(X x)_{i} \frac{\partial \varphi}{\partial x_{i}}(x),
$$

$\varphi \in C^{\infty}\left(\mathbf{R}^{n}\right), x \in \mathbf{R}^{n},(X x)_{i}$ the $i$ th component of $X x$. Let us regard $L$ as a linear mapping of $\mathscr{D}$ into itself, where $\mathscr{D}$ is the space of all $C^{\infty}$-functions with compact support on $\mathbf{R}^{n}$. Furthermore, we also regard $L$ as a linear mapping of $\mathscr{D}^{\prime}$ into itself defined in the usual way by continuous extension, where $\mathscr{D}^{\prime}$ is the dual space of $\mathscr{D}$, which is the space of (ordinary) distributions. The distributions annihilating the image $L \mathscr{D}$ of $L$ are just the distributions invariant under $e^{t X}, t \in \mathbf{R}$. We write $\mathscr{D}_{X}^{\prime}=(L \mathscr{D})^{\perp}$.

We ask the following questions closed related with each other:

(i) Is $L \mathscr{D}$ closed in $\mathscr{D}$ ?

(ii) How do we characterize the invariant distributions? Is there a canonical fundamental set?

(iii) Is the differential operator $L$ solvable in some sense? That means: When has the equation $L u=f$ a solution $u$ ?

Essentially this is a special case of the problem investigated in [9]. Nevertheless, it seems to be very difficult to answer these questions in general. (See the examples below.)

In [9], questions (i) and (ii) are studied in a more general framework: Let $M$ be a differentiable manifold and $\mathscr{L}$ a Lie algebra of infinitesimal transformations on $X$. Then the set $\operatorname{Div}(\mathscr{L})$ of all finite sums $\sum L_{i} \varphi_{i}$, where $L_{i} \in \mathscr{L}$ and $\varphi_{i} \in \mathscr{D}(M)$, is characterized in some special situations. Particularly, if at each point of $M$ the vector

Received by the editors December 8, 1983.

1980 Mathematics Subject Classification. Primary 58G99, 35A05, 35D05.

Key words and phrases. Divergences, invariant distributions, differential operators with critical points. 
fields in $\mathscr{L}$ give an $m$-dimensional subspace of the tangent space to $M$, it is claimed in [9, Theorem 2], that (under some additional assumptions)

(a) $\operatorname{Div}(\mathscr{L})$ is closed;

(b) $g \in \mathscr{D}(M)$ belongs to $\operatorname{Div}(\mathscr{L})$ iff $g$ is annihilated by the invariant measures on the integral manifolds.

In $[5, \S 4]$, a counterexample to (b) is given. (See also [1, §2.1] for new versions of [9, Theorem 2]; see [2-4].) In Example 2 we shall see that (a) is false, too.

Coming back to our special situation, we deal with the case that $X$ is a nilpotent matrix. In view of Example 2 we are suggested to work with $\mathscr{S}$ and $\mathscr{S}^{\prime}$ rather than with $\mathscr{D}$ and $\mathscr{D}^{\prime}$, where $\mathscr{S}$ is the Schwartz space of rapidly decreasing smooth functions and $\mathscr{S}^{\prime}$ its dual space, which is the space of tempered distributions. Working with $\mathscr{S}$ and $\mathscr{S}^{\prime}$ we get satisfactory answers to our questions.

Let us state the results: We call a pair $(v, w)$ of vectors in $\mathbf{R}^{n} X$-admissible if $v \neq 0$ and $X w \neq 0$. For $X$-admissible pairs $(v, w)$ and for integer $k \geqslant 0$ we define the tempered invariant distribution

$$
T_{v, w}^{(k)}(\varphi):=\int_{\mathbf{R}} \nabla_{v}^{k}\left(\varphi \circ e^{t X}\right)(w) d t, \quad \varphi \in \mathscr{S},
$$

where $\nabla_{t}$, denotes the directional derivative. We write $\mathscr{M}_{X}$ (resp. $\mathscr{M}_{X}^{\circ}$ ) for the set of all $T_{v^{\prime}, w}^{(k)}\left(\right.$ resp. $\left.T_{v^{\prime}, w}^{\left({ }^{\circ}\right)}\right)$. Clearly, the elements of $\mathscr{M}_{X}^{\circ}$ are just the invariant measures on the nontrivial $e^{\mathbf{R} X}$-orbits in $\mathbf{R}^{n}$.

THEOREM. Let $X$ be an arbitrary nonzero real nilpotent $(n \times n)$-matrix and let L be the infinitesimal transformation associated with $X$.

Then $L \mathscr{S}$ is closed in $\mathscr{S}$. Moreover the invariant tempered distributions can be characterized as follows:

For $\operatorname{rank}(X)=1$ the invariant orbital measures form a fundamental set. For $\operatorname{rank}(X)>1$ the set $\mathscr{M}_{X}$ is fundamental. (Note that in general the invariant orbital measures do not form a fundamental set according to $[5, \S 1]$.)

COROLLARY. The differential operator $L$ regarded as a mapping of $\mathscr{S}^{\prime}$ into itself is surjective.

To prove the theorem, Lemma 2.2 of [6] is crucially used. For the convenience of the reader it is cited here:

Lemma A. Suppose that $\mathbf{R}^{n-1} \hat{=}\left\{x \in \mathbf{R}^{n} \mid x_{1}=0\right\}$ is $X$-invariant and contains the kernel of $X$. Let $L^{\prime}$ be the infinitesimal transformation on $\mathbf{R}^{n-1}$ associated with the restriction of $X$ to $\mathbf{R}^{n-1}$. Let $\mathscr{M} \subseteq \mathscr{S}_{X}^{\prime}\left(\mathbf{R}^{n}\right)$ be a set of invariant tempered distributions containing the invariant measures on the orbits in $\left\{x_{1} \neq 0\right\}$ and satisfying the following conditions:

(i) if $\varphi \in \mathscr{S}\left(\mathbf{R}^{n}\right)$ and $x_{1} \varphi \in \mathscr{M}^{\perp}$, then $\varphi \in \mathscr{M}^{\perp}$;

(ii) if $\varphi \in \mathscr{M}^{\perp}$, then the restriction of $\varphi$ to $\mathbf{R}^{n-1}$ belongs to $L^{\prime} \mathscr{S}\left(\mathbf{R}^{n-1}\right)$. Then $\mathscr{M}^{\perp}=\operatorname{L} \mathscr{S}\left(\mathbf{R}^{n}\right)$.

2. Examples. To explain the area of validity of the assertions in the theorem we give two examples. Example 1 shows that the assertions do not need to be valid if $X$ is not nilpotent. In Example 2 we see that $\mathscr{S}$ cannot be replaced by $\mathscr{D}$ or $\mathscr{E}$. 
EXAmple 1. (Compare to Example 1 of [9].) Let $\alpha, \beta$ be real numbers with $\alpha / \beta$ irrational and let

$$
X=\left(\begin{array}{rrrr}
0 & -\alpha & 0 & 0 \\
\alpha & 0 & 0 & 0 \\
0 & 0 & 0 & -\beta \\
0 & 0 & \beta & 0
\end{array}\right)
$$

Then the one-parameter-subgroup $e^{t X}, t \in \mathbf{R}$, in $\mathrm{SO}(4)$ is not closed. By [8, Chapter IV, Theorem D], it is easily seen that the closure $\overline{L \mathscr{D}}$ of $L \mathscr{D}$ in $\mathscr{D}$ is just the set of all test functions $g$ for which $\int_{H} g(b x) d b=0$ for all $x \in \mathbf{R}^{4}$, where $H$ is the closure of $e^{\mathbf{R} X}$ in $\mathrm{SO}(4)$. Using a rotation invariant partition of unity we conclude that the closure $\overline{L \mathscr{S}}$ of $L \mathscr{S}$ in $\mathscr{S}$ is also the set of all $g \in \mathscr{S}$ for which $\int_{H} g(b x) d b=0$.

For $x \in \mathbf{R}^{4}$ we write $x=(y, z)$ where $y, z \in \mathbf{R}^{2}$. By polar decomposition we have $y=r \cdot e(\sigma)$ where $e(\sigma):=(\cos \sigma, \sin \sigma)$; similarly $z=s \cdot e(\tau)$. Let $\gamma$ be a test function on $] 0, \infty\left[\right.$ with $\gamma(1)=1$. If $h(y, z)$ is a $C^{\infty}$-function on $\left\{x \in \mathbf{R}^{4} \mid\right.$ $|y|=|z|=1\}$ for which $\int_{0}^{2 \pi} \int_{0}^{2 \pi} h(e(\sigma), e(\tau)) d \sigma d \tau=0$, then the function $g(x):=\gamma(r) \gamma(s) h(e(\sigma), e(\tau))$ belongs to $\mathscr{D}$ and satisfies the condition $\int_{H} g(b x) d b=0$ for all $x \in \mathbf{R}^{4}$. Assuming $g \in L \mathscr{D}$ or $g \in L \mathscr{S}$, say $g=L \varphi$, we receive

$$
h(e(\sigma), e(\tau))=\alpha \frac{\partial}{\partial \sigma} \Psi(e(\sigma), e(\tau))+\beta \frac{\partial}{\partial \tau} \Psi(e(\sigma), e(\tau))
$$

for the $C^{\infty}$-function $\Psi(y, z):=-\varphi(y, z)$ on $\{|y|=|z|=1\}$. But this is not possible for every $h$, when $\alpha / \beta$ is a Liouville number [9, Example 1]. Thus neither $L \mathscr{D}$ nor $L \mathscr{S}$ is closed in $\mathscr{D}$ and $\mathscr{S}$, respectively, whenever $\alpha / \beta$ is a Liouville number.

ExAMPle 2. Let

$$
X=\left(\begin{array}{lll}
0 & 0 & 0 \\
1 & 0 & 0 \\
0 & 1 & 0
\end{array}\right)
$$

It is shown in [5] that there are invariant tempered distributions on $\mathbf{R}^{3}$, which cannot be approximated by linear combinations of invariant orbital measures. More precisely, let $\alpha, \beta, \gamma \in \mathscr{D}(\mathbf{R})$ such that $\alpha(0)=0$ and $\beta(-t)=-\beta(t)$ for $t \in \mathbf{R}$ and put $g(x):=\alpha\left(x_{1}\right) \beta\left(x_{2}\right) \gamma\left(x_{3}\right) \in \mathscr{D}\left(\mathbf{R}^{3}\right)$. Then $g$ is annihilated by all invariant orbital measures, but if $k>0$ we have $T_{v, w}^{(k)}(g) \neq 0$ for $\alpha=x_{1}^{k} \eta, \eta \in \mathscr{D}(\mathbf{R})$, such that $\eta(0) \neq 0$, and for suitable functions $\beta, \gamma$, where $v=(1,0,0)$ and $w=(0,1,0)$. Therefore, assertion (b) of Theorem 2 in [9] (see introduction) cannot be valid. However, at this point it is not yet clear if $L \mathscr{D}$ is closed or not.

To answer this question we select $\alpha, \beta, \gamma$ such that $\beta \geqslant 0$ on $] 0, \infty[, \beta(1)>0$, $\gamma \geqslant 0, \gamma(0)>0, \alpha>0$ on the open interval $] 0,1[$ and $\alpha=0$ outside of $] 0,1[$. Now we find a sequence $\left(\alpha_{\nu}\right)$ converging to $\alpha$ in $\mathscr{D}(\mathbf{R})$ with $\alpha_{\nu}=0$ outside of $] \varepsilon_{\nu}, 1$, $\varepsilon_{\nu}>0$. Then the sequence $g_{\nu}(x):=\alpha_{\nu}\left(x_{1}\right) \beta\left(x_{2}\right) \gamma\left(x_{3}\right)$ converges to $g(x):=$ $\alpha\left(x_{1}\right) \beta\left(x_{2}\right) \gamma\left(x_{3}\right)$ in $\mathscr{D}\left(\mathbf{R}^{3}\right)$. At first we show that $g_{\nu} \in L \mathscr{D}$ for all $\nu$. By [6, Lemma 2.6], $g_{\nu}$ belongs to $L \mathscr{S}$. The function $\varphi_{\nu} \in \mathscr{S}$ for which $g_{\nu}=L \varphi_{\nu}$ is obtained by the formula

$$
\varphi_{\nu}(x)=\int_{0}^{\infty} g_{\nu}\left(x_{1}, t x_{1}+x_{2}, \frac{t^{2}}{2} x_{1}+t x_{2}+x_{3}\right) d t
$$


(See [6, §2.1].) Using this formula, the fact that $\varphi_{\nu}$ has compact support is verified by a routine calculation keeping in mind that $g_{\nu}(x)=0$ for $x_{1}<\varepsilon_{\nu}$. From $g_{\nu} \in L \mathscr{D}$ we receive $g \in \overline{L \mathscr{D}}$. Assuming $g \in L \mathscr{D}$, say $g=L \varphi$, our formula implies

$$
\varphi\left(\varepsilon, 0,-\frac{1}{2 \varepsilon}\right)=\alpha(\varepsilon) \int_{0}^{\infty} \beta(t \varepsilon) \gamma\left(\frac{t^{2}}{2} \varepsilon-\frac{1}{2 \varepsilon}\right) d t>0
$$

for all $0<\varepsilon<1$ because the integrand is $\geqslant 0$ and $>0$ for $t=1 / \varepsilon$. But this is not possible, since $\varphi$ has compact support. Thus $L \mathscr{D}$ is not closed in $\mathscr{D}$.

Choosing $\beta$ in such a way that $\beta=0$ on a neighbourhood of 0 we get a counterexample to assertion (a) of Theorem 2 in [9] (see introduction).

Moreover, it follows by functional analysis that the equation $L u=f$ does not have a solution $u \in \mathscr{D}^{\prime}$ for every $f \in \mathscr{D}^{\prime}$.

Furthermore, we can conclude that $L \mathscr{E}$ is not closed in $\mathscr{E}$, where $\mathscr{E}$ is the space of all infinitely differentiable functions provided with the usual topology. Suppose that $L \mathscr{E}$ is closed in $\mathscr{E}$. Then the mapping $L: \mathscr{E}^{\prime} \rightarrow \mathscr{E}^{\prime}$ has a closed range. Therefore we can find a distribution $u$ with compact support satisfying $L u=g$. Applying once more [6, Lemma 2.6], we receive $L \varphi=g$ for the function $\varphi \in \mathscr{S}$ given by the previous formula. It follows that $L(\varphi-u)=0$, therefore the distribution $\varphi-u$ is invariant. We determine $r \geqslant 2$ such that the support of $u$ is contained in $\left\{\left|x_{i}\right| \leqslant r\right.$, $i=1,2,3\}$. Now let $\varepsilon<1 / 2 r$ and select a test function $\Psi \geqslant 0$ satisfying $\Psi(\varepsilon, 0,-1 / 2 \varepsilon)>0$ such that the support of $\Psi$ is contained in a $\delta$-neighbourhood of $(\varepsilon, 0,-1 / 2 \varepsilon)$, where $\delta$ is a sufficiently small positive number, $\delta<\varepsilon / 2$. For $t>$ $2(r+1) / \varepsilon$ the support of $\Psi \circ e^{-t X}$ is contained in $\left\{x_{1}>\varepsilon / 2, x_{2}>r\right\}$, therefore we have

$$
\langle u, \Psi\rangle=\left\langle u, \Psi \circ e^{-t X}\right\rangle=0 \text { and }\left\langle\varphi, \Psi \circ e^{-t X}\right\rangle=0
$$

but $\langle\varphi, \Psi\rangle \neq 0$. Using the invariance of $\varphi-u$ we get the following contradiction:

$$
0 \neq\langle\varphi, \Psi\rangle=\langle\varphi-u, \Psi\rangle=\left\langle\varphi-u, \Psi \circ e^{-t X}\right\rangle=0
$$

3. Proofs. Let $X$ be an arbitrary nilpotent $(n \times n)$-matrix, $X \neq 0$. After change of basis we may assume that $X$ has the form

$$
\left(\begin{array}{ccccc}
0 & \ddots & & & \\
1 & \ddots & & & \\
& \varepsilon_{2} & \ddots & & \\
& & \ddots & \ddots & \\
& & & \varepsilon_{n-1} & 0
\end{array}\right)
$$

where $\varepsilon_{j} \in\{0,1\}, j=2, \ldots, n-1$.

Lemma 1. Let $\varphi \in \mathscr{S}$. Assume that $x_{1} \varphi$ is annihilated by all $T \in \mathscr{M}_{X}$. (We write $\left.x_{1} \varphi \in \mathscr{M}_{X}^{\perp}.\right)$ Then $T_{v, w}^{(k)}(\varphi)=0$ whenever $v_{1} \neq 0\left(v_{1}=\right.$ the first component of $\left.v\right)$.

Proof. By [6, §2.4], we have the formula $x_{1} T_{v, w}^{(k)}=k v_{1} T_{v, w}^{(k-1)}+w_{1} T_{v, w}^{(k)}, k>0$. Therefore $k v_{1} T_{v, w}^{(k-1)}(\varphi)+w_{1} T_{v, w}^{(k)}(\varphi)=0$. For $w_{1}=0$ the assertion follows immediately. For $w_{1} \neq 0$ we conclude $T_{v, w}^{(\circ)}(\varphi)=0$ from $0=\left\langle T_{v, w}^{(\circ)}, x_{1} \varphi\right\rangle=$ $w_{1}\left\langle T_{v, w}^{(\circ)}, \varphi\right\rangle$ and proceed by induction on $k$. 
LemMa 2. Let $\varphi \in \mathscr{S}$. If $x_{1} \varphi \in \mathscr{M}_{X}^{\perp}$ then $\varphi \in \mathscr{M}_{X}^{\perp}$.

Proof. By Lemma 1, we have only to prove that $T_{v, w}^{(k)}(\varphi)=0$ whenever $v_{1}=0$. Now, by Lemma 1,

$$
0=T_{v^{(v)}, w}^{(k)}(\varphi)=\int \nabla_{v^{(\nu)}}^{k}\left(\varphi \circ e^{t X}\right)(w) d t
$$

where $v^{(\nu)}=v+(1 / \nu, 0, \ldots, 0), \nu \in \mathbf{N}$. Using the formula

$$
\nabla_{v^{(\nu)}}^{k}=\sum_{m=0}^{k}\left(\begin{array}{c}
k \\
m
\end{array}\right) \frac{1}{\nu^{m}} \frac{\partial^{m}}{\partial x_{1}^{m}} \nabla_{v}^{k-m}
$$

we conclude

$$
0=\sum_{m=0}^{k}\left(\begin{array}{l}
k \\
m
\end{array}\right) \frac{1}{\nu^{m}} \int \frac{\partial^{m}}{\partial x_{1}^{m}} \nabla_{v}^{k-m}\left(\varphi \circ e^{t X}\right)(w) d t
$$

For $\nu \rightarrow \infty$ we get

$$
0=\int \nabla_{v}^{k}\left(\varphi \circ e^{t X}\right)(w) d t=T_{v, w}^{(k)}(\varphi)
$$

Lemma 3. Let the rank of $X$ be equal to 1 . Let $\varphi \in \mathscr{S}$. Suppose that $\varphi \in\left(\mathscr{M}_{X}^{\circ}\right)^{\perp}$ and $\varphi(x)=0$ whenever $x_{1}=0$. Then $\varphi \in L \mathscr{S}$.

Proof. Compare to [6, Lemma 2.5]. We get $\varphi=x_{1} \chi$ where $\chi \in\left(\mathscr{M}_{X}^{\circ}\right)^{\perp}$. Therefore $\int_{\mathbf{R}} \chi(x) d x_{2}=0$. Thus there is a function $\psi \in \mathscr{S}_{\text {such that } \chi} \chi \partial \psi / \partial x_{2}$. We get $\varphi=x_{1} \partial \psi / \partial x_{2}=-L \psi$.

LEMMA 4. Let the rank of $X$ be equal to 2 and let $L^{\prime}$ be the infinitesimal transformation on $\mathbf{R}^{n-1} \stackrel{\wedge}{=}\left\{x \in \mathbf{R}^{n} \mid x_{1}=0\right\}$ associated with the matrix

$$
\left(\begin{array}{ccccc}
0 & \ddots & & & \\
\varepsilon_{2} & \ddots & & & \\
& \ddots & \ddots & \ddots & \\
& & \varepsilon_{n-1} & & 0
\end{array}\right)
$$

Then for every $\varphi \in\left(\mathscr{M}_{X}^{\circ}\right)^{\perp}$ the restriction $\varphi^{\prime}$ to $\mathbf{R}^{n-1}$ belongs to $L^{\prime} \mathscr{S}\left(\mathbf{R}^{n-1}\right)$.

Proof. Obviously it is sufficient to consider the two cases $\varepsilon_{j}=\delta_{2, j}$ and $\varepsilon_{j}=\delta_{3, j}$, $j=2, \ldots, n-1$.

Let $\varepsilon_{j}=\delta_{2, j}$. By Lemma 3, we have only to prove that $\varphi^{\prime}\left(x_{2}, x_{3}, \ldots, x_{n}\right)=0$ whenever $x_{2}=0$. By assumption, for all $\nu \in \mathbf{N}$,

$$
0=\int \varphi\left(e^{t X}\left(\frac{1}{\nu^{2}}, 0, x_{3}, \ldots, x_{n}\right)\right) d t=\nu \int \varphi\left(\frac{1}{\nu^{2}}, \frac{t}{\nu}, \frac{t^{2}}{2}+x_{3}, x_{4}, \ldots, x_{n}\right) d t .
$$

For $\nu \rightarrow \infty$ we get $\int \varphi\left(0,0, t^{2} / 2+x_{3}, \ldots, x_{n}\right) d t=0$ for all $x_{3}, \ldots, x_{n}$. It is proved in [6, Lemma 2.6] that from this it follows that $\varphi\left(0,0, x_{3}, \ldots, x_{n}\right)=0$ for all $x_{3}, \ldots, x_{n}$. 
Let $\varepsilon_{j}=\delta_{3, j}$. By Lemma 3, we have only to prove that $\varphi^{\prime}\left(x_{2}, x_{3}, \ldots, x_{n}\right)=0$ whenever $x_{3}=0$. By assumption, for all $\nu \in \mathbf{N}$ and for every nonzero vector $z=\left(z_{1}, z_{2}\right) \in \mathbf{R}^{2}$ we have

$$
\begin{aligned}
0 & =\int \varphi\left(e^{t X}\left(\frac{z_{1}}{\nu}, x_{2}, \frac{z_{2}}{\nu}, x_{4}, \ldots, x_{n}\right)\right) d t \\
& =\nu \int \varphi\left(\frac{z_{1}}{\nu}, x_{2}+t z_{1}, \frac{z_{2}}{\nu}, x_{4}+t z_{2}, \ldots, x_{n}\right) d t .
\end{aligned}
$$

For $\nu \rightarrow \infty$ we get $\int \varphi\left(0, x_{2}+t z_{2}, 0, x_{4}+t z_{2}, \ldots, x_{n}\right) d t=0$ for all $x_{2}, x_{4}, \ldots, x_{n}$. This means that the one-dimensional Radon transform of the function $\left(x_{2}, x_{4}\right) \rightarrow$ $\varphi\left(0, x_{2}, 0, x_{4}, \ldots, x_{n}\right)$ is identically 0 for all $x_{5}, \ldots, x_{n}$. Now the assertion follows. (See [7, Chapter I, §6].)

Proof of The Theorem. For $\operatorname{rank}(X)=1$ the Theorem is just Lemma 3. For $\operatorname{rank}(X)>1$ we prove $\mathscr{M}_{X}^{\perp}=L \mathscr{S}$ by induction on $\operatorname{rank}(X)$. For $\operatorname{rank}(X)=2$ the assertion follows from Lemma $\mathrm{A}$, using Lemmas 2 and 4 . For $\operatorname{rank}(X)>2$ the assertion follows from Lemma A, using Lemma 2 and the induction hypothesis.

Proof of The Corollary. By the Theorem, we only have to prove that $L: \mathscr{S} \rightarrow \mathscr{S}$ is injective. Now, if $L \varphi=0$ for $\varphi \in \mathscr{S}$, then $\varphi$ must be invariant because of $\mathscr{S}_{X}^{\prime}=(L \mathscr{S})^{\perp}$; i.e. $\varphi$ is constant on the orbits. In view of the fact that "almost all" orbits are unbounded this is not possible except for $\varphi=0$.

\section{REFERENCES}

1. R. Barrà, Divergences et distributions invariantes, Thèse de $3^{e}$ cycle, $n^{\circ} 723$, Université de Poitiers, 1978.

2. Fonctions divergences et distributions invariantes, Bull. Sci. Math. (2) 105 (1981), 49-71.

3. __ Fonctions divergences et distributions invariantes. II, Bull. Sci. Math. (2) 107 (1983), 209-217.

4. Distributions invariantes. III, Preprint.

5. J. Dixmier, Sur les distributions invariantes par un groupe nilpotent, C. R. Acad. Sci. Paris Ser. A 285 (1977), 7-10.

6. R. Felix, Das Syntheseproblem für invariante Distributionen, Invent. Math. 65 (1981), 85-96.

7. S. Helgason, The Radon transform, Progress Math. 5, Birkhäuser, Boston-Basel-Stuttgart, 1980.

8. C. S. Herz, Analyse harmonique à plusieurs variables, Publ. Math. Orsay, 1965.

9. __ Functions which are divergences, Amer. J. Math. 92 (1970), 641-656.

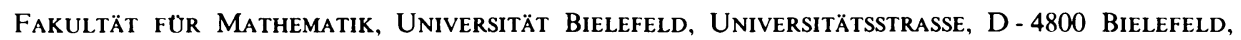
WEST GERMANY 